\title{
Analytical Solution for the Combined Solar Radiation Pressure and Luni- Solar Effects on the Orbits of High Altitude Satellites
}

\author{
Nadia A. Saad ${ }^{1, *}$, Kh. I. Khalil ${ }^{2}$ and Magdy Y. Amin ${ }^{3}$ \\ ${ }^{1,2}$ National Research Institute of Astronomy and Geophysics, Helwan, Cairo, Egypt \\ ${ }^{3}$ Astronomy and Astrochemistry, Department Astronomy, Faculty of Science, Cairo University, Egypt
}

\begin{abstract}
A new analytical solution is obtained for the motion of high-altitude earth satellites. The basic idea is to study the joint effects of direct solar radiation pressure and the gravitation of the Earth, Moon and the Sun on the orbits of the satellites. The mathematical model includes the zonal harmonics of the geopotential effects up to $J_{4}$. The order of magnitude of each perturbing term is assessed. The formulae for the all perturbations forces are obtained up to the fourth order (where the mean motion of the moon $\left(\sim 10^{-2}\right)$ is considered a small quantity of first order). The short, intermediate and long-period terms are eliminated from the Hamiltonian using a perturbation technique based on the Lie-Deprit-Kamel transform through three canonical transformations. The solution is intended to be used for ephemeris predictions for orbiters whose orbital semimajor axes are in the range of 15000 to $70000 \mathrm{kms}$.
\end{abstract}

Key Words: Astrodynamics, artificial satellites theories, Hamiltonian system, Lie-Deprit-Kamel transform, solar radiation pressure, luni- solar effects.

\section{INTRODUCTION}

The study of the effect of direct solar radiation pressure on the orbits of the artificial satellites was discussed in a lot of literatures, starting from pioneering works of [1-4] and other of [5-9]. Also the theory of third body perturbations on an artificial satellite motion was developed by many authors in the past. The formulae for secular and periodic perturbations in orbital elements were obtained with the use of different methods [10-17]. But the joint effects of radiation pressure and the gravitational attraction of the Earth, Moon and Sun are rarely mentioned in the literature.

Musen (1960) [1] derived first order expressions for the rates of change in the osculating elements caused by the direct solar radiation pressure. He used the method of variation of vector elements.

Kaula (1962) [18] just developed the Lunar and Solar disturbing function for a close satellite and developed a quasi potential for the radiation pressure effects for use it in the equation of motion. He did not obtain the solution.

Sehnal (1975) [19] discussed the direct solar radiation pressure, as one of the non-gravitational forces, from all its different aspects.

Anselmo et al. (1983) [20] had analyzed the perturbations due to solar radiation pressure, only, on the orbit of a high artificial satellite. The latter was modeled in a simplified way (axisymmetric body), which seems suitable to describe the main effects for existing telecommunication satellites. He used the regularized general perturbation equations,

*Address correspondence to this author at the National Research Institute of Astronomy and Geophysics, Helwan, Cairo, Egypt; Tel: +2025541100; Fax:+2025548020; E-mail:drnadia@nriag.sci.eg. by expressing the force in the moving Gauss' reference frame and by expanding the results in terms of some small parameters, referring both to the orbit (small eccentricity and inclination) and to the spacecraft's attitude. He used the general perturbation equations in Gauss' form and he neglected the oblateness of the Earth.

The present work considers the motion of an earth satellite orbiting at a high altitude in an orbit with semimajor axis in the range of 15000 to $70000 \mathrm{~km}$ at low inclination $I \leq 10^{\circ}$, with no restriction on the orbital eccentricity. The mathematical model includes the non sphericity of the earth's gravitational field up to $J_{4}$ besides the effects of the lunar and solar gravitational attractions and solar radiation pressure. The disturbing function is developed completely apart from considerations of order [21]. Then the orders are assigned such that the terms are retained whenever their contributions are of fourth order, where the mean motion of the moon $\left(\sim 10^{-2}\right)$ is considered small quantity of the first order. The Hamiltonian has been formed in terms of a set of ten canonical elements representing the Delaunay variables augmented by the arguments of latitude of the moon and sun and their conjugate momenta. Then the short, intermediate and long-period terms are eliminated from the Hamiltonian with analytical perturbation technique based on the LieDeprit-Kamel transform [22, 23].

Finally a procedure for the computation of the position and velocity at any time is presented.

\section{THE FORCE FUNCTION}

\subsection{The Force Functions Due to the Gravitational Effects}

Firstly we derive the equations of motion of the satellite under the gravitation effects of the earth (as primary), and the moon and sun (as perturbations). Let the subscripts 0,1 , 2, 3 refer respectively to the earth, satellite, moon, and sun. 
The equations of motion of the four point masses $m_{i}$ in an inertial frame are

$$
m_{i} \ddot{\bar{\rho}}_{i}=\tilde{\nabla}_{\rho_{i}} U \quad(i=0,1,2,3)
$$

where $U$ is the force function given by

$$
U=k^{2} \sum_{j>i}^{3} \frac{m_{i} m_{j}}{r_{i j}} \quad(i, j=0, \ldots 3)
$$

$\bar{\rho}_{i}=\left(\xi_{i}, \eta_{i}, \xi_{i}\right)$ is the position vector of anyone of the four bodies with respect to the inertial frame and $r_{i j}$ is the distance between the mass points

It is convenient to refer the satellite and the moon to the earth and the sun to the centre of mass of the earth-moon system. Denoting the new position vectors by $\bar{r}_{i}$, we have

$$
\begin{gathered}
\overline{r_{1}}=\bar{\rho}_{1}-\bar{\rho}_{0} \\
\overline{r_{2}}=\bar{\rho}_{2}-\bar{\rho}_{0} \\
\overline{r_{3}}=\bar{\rho}_{3}-\frac{m_{0} \bar{\rho}_{0}+m_{2} \bar{\rho}_{2}}{m_{0}+m_{2}}
\end{gathered}
$$

The equations of motion (1) must be transformed accordingly where the partials in Eq.(1) transform as:

$$
\frac{\partial U}{\partial \bar{\rho}_{j}}=\sum_{k=1}^{3} \frac{\partial U}{\partial \bar{r}_{k}} \frac{\partial \bar{r}_{k}}{\partial \bar{\rho}_{j}}, \quad(j=0,1,2,3)
$$

Resulting in

$$
\begin{aligned}
& \tilde{\nabla}_{0} U=-\nabla_{1} U-\nabla_{2} U-\frac{m_{0}}{m_{0}+m_{2}} \nabla_{3} U, \\
& \tilde{\nabla}_{1} U=\nabla_{1} U \\
& \tilde{\nabla}_{2} U=\nabla_{2} U-\frac{m_{2}}{m_{0}+m_{2}} \nabla_{3} U, \quad \tilde{\nabla}_{3} U=\nabla_{3} U
\end{aligned}
$$

where $\nabla \equiv\left(\frac{\partial}{\partial x}, \frac{\partial}{\partial y}, \frac{\partial}{\partial z}\right)^{T}, \tilde{\nabla} \equiv\left(\frac{\partial}{\partial \xi}, \frac{\partial}{\partial \eta}, \frac{\partial}{\partial \xi}\right)^{T}$ Making use of

Eqs (1), (2.1), and (2.2)

the equations of motion of $m_{1}$ can be written as:

$$
\ddot{\bar{r}_{1}}=\frac{m_{0}+m_{1}}{m_{0} m_{1}} \nabla_{1} U+\frac{1}{m_{0}} \nabla_{2} U+\frac{1}{m_{0}+m_{2}} \nabla_{3} U
$$

In order to express $U$ in terms of the new variables, we first choose the centre of mass of the entire system as origin of the inertial frame, with this choice $\sum_{j=0}^{3} m_{j} \bar{\rho}_{j}=0$

Combining Eq. (2.1) and Eq. (4) then:

$$
\begin{aligned}
& \bar{\rho}_{0}=-\frac{m_{2}}{m_{0}+m_{2}} \bar{r}_{2}-\frac{m_{3}}{m_{0}+m_{2}+m_{3}} \bar{r}_{3}, \\
& \bar{\rho}_{1}=\bar{r}_{1}-\frac{m_{2}}{m_{0}+m_{2}} \bar{r}_{2}-\frac{m_{3}}{m_{0}+m_{2}+m_{3}} \bar{r}_{3} \\
& \bar{\rho}_{2}=\frac{m_{0}}{m_{0}+m_{2}} \bar{r}_{2}-\frac{m_{3}}{m_{0}+m_{2}+m_{3}} \bar{r}_{3}, \quad \bar{\rho}_{3}=\frac{m_{0}+m_{2}}{m_{0}+m_{2}+m_{3}} \bar{r}_{3}
\end{aligned}
$$

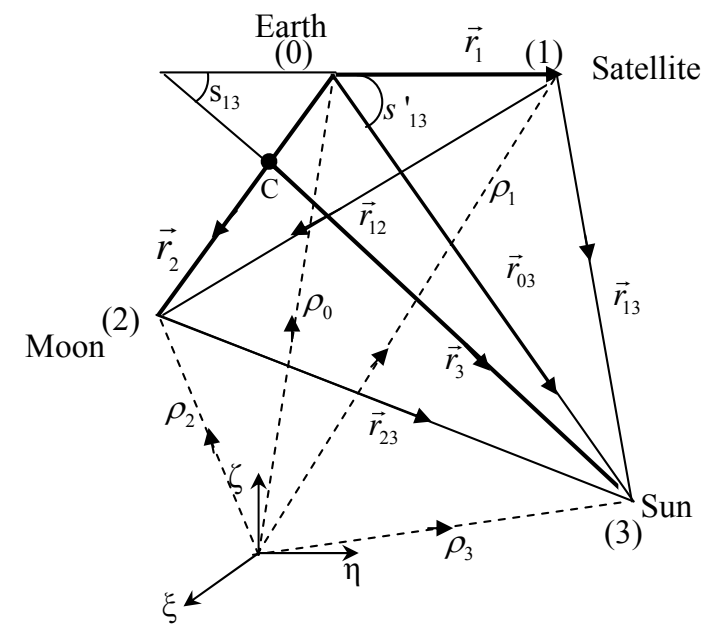

Fig. (1). Relative positions of the earth, satellite, moon and sun.

$$
\begin{aligned}
& \text { appealing now to the relations } \\
& r_{i j}^{2}=\left(\xi_{j}-\xi_{i}\right)^{2}+\left(\eta_{j}-\eta_{i}\right)^{2}+\left(\xi_{j}-\xi_{i}\right)^{2} \\
& \text { and from Fig. (1), } \quad r_{01}^{2}=r_{1}^{2}, \quad r_{02}^{2}=r_{2}^{2} \\
& r_{03}^{2}=r_{3}^{2}+\frac{2 m_{2}}{m_{0}+m_{2}} \bar{r}_{2} \cdot \bar{r}_{3}+\left(\frac{m_{2}}{m_{0}+m_{2}}\right)^{2} r_{2}^{2} \\
& r_{2}^{2}=r^{2}-2 \bar{r} \cdot \bar{r}_{2}+r_{2}^{2} \\
& r_{13}^{2}=r_{3}^{2}+\frac{2 m_{2}}{m_{0}+m_{2}} \bar{r}_{2} \cdot \bar{r}_{3}+\left(\frac{m_{2}}{m_{0}+m_{2}}\right)^{2} r_{2}^{2}-2 \bar{r}_{1} \cdot \bar{r}_{3}-\frac{2 m_{2}}{m_{0}+m_{2}} \bar{r}_{1} \cdot \bar{r}_{2}+r_{1}^{2} \\
& r_{23}^{2}=r_{3}^{2}-\frac{2 m_{0}}{m_{0}+m_{2}} \bar{r}_{2} \cdot \bar{r}_{3}+\left(\frac{m_{0}}{m_{0}+m_{2}}\right)^{2} r_{2}^{2}
\end{aligned}
$$

with these relations we can express $U$ in terms of the new coordinates.

Next, we have to express the partials in Eq. (3) explicitly in terms of the new coordinates. In doing so we make use of the relation: $\nabla \frac{1}{r}=-\frac{\bar{r}}{r^{3}} \nabla \bar{r}$

which leads to the following, required partial derivatives

$$
\begin{aligned}
& \nabla_{1} \frac{1}{r_{1}}=-\frac{\bar{r}_{1}}{r_{1}^{3}}, \\
& \nabla_{1} \frac{1}{r_{12}}=\frac{\bar{r}_{2}-\bar{r}_{1}}{r_{12}^{3}}, \quad \frac{)}{r_{3}}=\frac{)}{r_{3}^{3}}\left(\bar{r} \frac{m_{0}}{m_{1}+m_{0}} \bar{r}_{0} \bar{r}_{3}\right), \\
& \nabla_{2} \frac{1}{r_{2}}=-\frac{\bar{r}_{2}}{r_{2}^{3}} \\
& \nabla_{2} \frac{1}{r_{03}}=-\frac{m_{2}}{m_{0}+m_{2}} \frac{1}{r_{03}^{3}}\left(\bar{r}_{3}+\frac{m_{2}}{m_{0}+m_{2}} \bar{r}_{2}\right), \\
& \nabla_{2} \frac{1}{r_{23}}=\frac{m_{0}}{m_{0}+m_{2}} \frac{1}{r_{23}^{3}}\left(\bar{r}_{3}-\frac{m_{0}}{m_{0}+m_{2}} \bar{r}_{2}\right)
\end{aligned}
$$




$$
\begin{aligned}
& 3 \frac{1}{r_{03}}=\frac{1}{r_{03}^{3}} \bar{r}_{3}+\frac{m_{2}}{m_{0}+m_{2}} \bar{r}_{2}(, \\
& 3 \frac{1}{r_{33}}=\frac{1}{r_{) 3}^{3}}\left(\bar{r}_{3} \frac{m_{(}}{m_{(}+m_{)}} \bar{r}_{)}\right)
\end{aligned}
$$

Substituting these derivatives into Eq. (3) it follows, after some simplifications, that:

$$
\begin{aligned}
& \ddot{\bar{r}}_{1}=k^{2} m_{0} \frac{\bar{r}_{1}}{r_{1}^{3}}+m_{2} \frac{\bar{r}_{2}}{r_{2}^{3}}+m_{2} \frac{\bar{r}_{1} \bar{r}_{2}}{r_{12}^{3}}+\frac{m_{3}}{r_{13}^{3}} \bar{r}_{1} \frac{m_{2}}{m_{0}+m_{2}} \bar{r}_{2} \bar{r}_{3}+ \\
& \left.\frac{m_{3}}{r_{03}^{3}}\left(\bar{r}_{3}+\frac{m_{2}}{m_{0}+m_{2}} \bar{r}_{2}\right)\right]
\end{aligned}
$$

This may be, better, written as $\ddot{\bar{r}_{1}}+{ }_{1} U_{\text {grav. }}+\frac{\mu}{\bar{r}_{1}}$,

where

$$
U_{\text {grav }}=k^{2}\left[\frac{m_{2}}{r_{12}}+\frac{m_{3}}{r_{13}} \quad \frac{m_{2}}{r_{2}^{3}} \bar{r}_{1} \cdot \bar{r}_{2} \quad \frac{m_{3}}{r_{03}^{3}}\left(\frac{m_{2}}{m_{0}+m_{2}} \bar{r}_{1} \cdot \bar{r}_{2}+\bar{r}_{1} \cdot \bar{r}_{3}\right)\right]
$$

$\mu \mu k^{2} m_{0}$ and $U_{\text {grav. }}$ represents the disturbing function due to the attractions of the moon and of the sun. In the following subsections we develop $U_{\text {grav }}$ in forms suitable to any subsequent analysis.

\subsection{Development of the Gravitational Disturbing Function}

The first step in the development of the disturbing function requires the expansion of $1 / r_{12}, 1 / r_{13}$, and $1 / r_{03}^{3}$ in series in the ratios $r_{1} / r_{2}, r_{1} / r_{3}$ and $r_{2} / r_{3}$ and in terms of three angles $s_{12}, s_{13}$ and $s_{23}$ where $\cos S_{i j}=\bar{r}_{i} \cdot \bar{r}_{j} / r_{i} r_{j}$. Then

$$
\begin{aligned}
& \frac{1}{r_{12}}+\frac{1}{r_{2}}\left[1 \quad 2 \frac{r_{1}}{r_{2}} \cos s_{12}+\left(\frac{r_{1}}{r_{2}}\right)^{2}\right]^{1 / 2} \\
& \left.\frac{1}{r_{13}}=\frac{1}{r_{3}}\left[1+\frac{) m_{)}}{m_{(}+m_{)}} \frac{r_{2}}{r_{3}} 2 \cos \right)_{3}+\left(\frac{m_{1}}{m_{(}+m_{)}}\right)^{)} \frac{r_{)}^{)}}{r_{3}^{)}}-\right) \frac{r_{1}}{r_{3}} 2 \cos s_{13} \\
& \left.\quad-\frac{) m_{)}}{m_{(}+m_{)}} \frac{r_{1}}{r_{3}} \frac{r_{3}}{r_{3}} 2 \cos s_{1)}+\frac{r_{1}^{)}}{r_{3}^{)}}\right]^{-1 /)} \\
& \frac{1}{r_{03}}+\frac{1}{r_{3}} 1+2 \frac{m_{2}}{m_{0}+m_{2}} \frac{r_{2}}{r_{3}} \cos s_{23}+\frac{m_{2}}{m_{0}+m_{2}} \frac{r_{2}^{2}}{r_{3}^{2}}
\end{aligned}
$$

Clearly the first and third of these may be expanded with the aid of Legendre polynomials, while for the second a binomial expansion will serve, so that:

$$
\begin{aligned}
& \frac{1}{r_{12}}=\frac{1}{r_{2}} \sum_{n \geq 0}\left(\frac{r_{1}}{r_{2}}\right)^{n} p_{n}\left(\cos s_{12}\right) \\
& \frac{1}{r_{03}}=\frac{1}{r_{3}}{ }_{n 0}(1)^{n} \frac{m_{2}}{m_{0}+m_{2}} \frac{r_{2}}{r_{3}} p_{n}\left(\cos s_{23}\right)
\end{aligned}
$$

$$
\frac{1}{r_{13}}=\frac{1}{r_{3}} 1 \frac{1}{2} X+\frac{3}{8} X^{2} \quad \frac{5}{16} X^{3}+\frac{35}{128} X^{4} \quad \ldots
$$

where

$$
\begin{aligned}
& X=2 \frac{m_{2}}{m_{0}+m_{2}} \frac{r_{2}}{r_{3}} \cos s_{23}+\frac{m_{2}}{m_{0}+m_{2}} \frac{r_{2}^{2}}{r_{3}^{2}} 2 \frac{r_{1}}{r_{3}} \cos s_{13} \\
& 2 \frac{m_{2}}{m_{0}+m_{2}} \frac{r_{1}}{r_{3}} \frac{r_{2}}{r_{3}} \cos s_{12}+\frac{r_{1}^{2}}{r_{3}^{2}}
\end{aligned}
$$

In order to determine the truncation point for each series we evaluate the coefficients and retaining terms with numerical values up to $10^{-8}$ then the above expressions yield

$$
\begin{aligned}
& \frac{1}{r_{12}}=\frac{1}{r_{2}}+\frac{r_{1}}{r_{2}^{2}} \cos s_{12}+\frac{r_{1}^{2}}{2 r_{2}^{3}}\left(3 \cos ^{2} s_{12} \quad 1\right)+\frac{r_{1}^{3}}{2 r_{2}^{4}}\left(5 \cos ^{3} s_{12}\right. \\
& \left.3 \cos s_{12}\right)+\frac{r_{1}^{4}}{8 r_{2}^{5}}\left(35 \cos ^{4} s_{12} \quad 30 \cos ^{2} s_{12}+3\right) \\
& \frac{1}{r_{03}^{3}}=\frac{1}{r_{3}^{3}} 3 M \frac{r_{2}}{r_{3}^{4}} \cos s_{23} \\
& \frac{1}{r_{13}}=\frac{1}{r_{3}}-M \frac{r_{2}}{r_{3}^{2}} \cos s_{23}+\frac{r_{1}}{r_{3}^{2}} \cos s_{13}+M \frac{r_{1} r_{2}}{r_{3}^{3}} \cos s_{12} \\
& -\frac{1}{2} M^{2} \frac{r_{2}^{2}}{r_{3}^{3}}-\frac{r_{1}^{2}}{2 r_{3}^{3}}+\frac{3}{2} M^{2} \frac{r_{2}^{2}}{r_{3}^{3}} \cos ^{2} s_{23} \\
& +\frac{3 r_{1}^{2}}{2 r_{3}^{3}} \cos ^{2} s_{13}-3 M \frac{r_{1} r_{2}}{r_{3}^{3}} \cos s_{13} \cos s_{23}+3 M \frac{r_{1}^{2} r_{2}}{r_{3}^{4}} \cos s_{12} \cos s_{13}
\end{aligned}
$$

where $M=m_{2} /\left(m_{0}+m_{2}\right)$. Substituting into Eq. (5), retaining terms up to $10^{8}$ we obtain

$$
\begin{aligned}
& U_{\text {grav. }}=k^{2}\left[\frac{m_{2}}{2} \frac{r_{1}^{2}}{r_{2}^{3}}\left(3 \cos ^{2} r_{12}-1\right)+\right. \\
& \left.\frac{m_{2}}{2} \frac{r_{1}^{3}}{r_{2}^{4}}\left(5 \cos ^{3} r_{12}-3 \cos r_{12}\right)+\frac{m_{3}}{2} \frac{r_{1}^{2}}{r_{3}^{3}}\left(3 \cos ^{2} r_{13}-1\right)\right]
\end{aligned}
$$

The next step is to express $\cos s_{12}$ and $\cos s_{13}$ in terms of the orbital elements of the orbiter, the moon, the earth and the sun. It is readily clear from Fig. (2) that the base vectors along $\bar{r}_{A}$ and $\bar{r}_{B}$ may be written as:

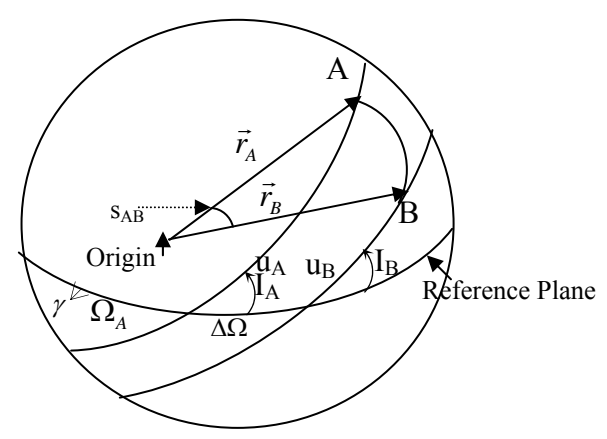

Fig. (2). Geometric relation between the angle $S_{A B}$ and the orbital elements of any two orbits A and B. 


$$
\begin{aligned}
& \hat{r}_{A}=\left(\begin{array}{c}
\cos u_{A} \\
\cos { }_{A} \operatorname{ss}_{A} \\
\operatorname{ss}_{A} \operatorname{ss}_{A} u_{A}
\end{array}\right)
\end{aligned}
$$

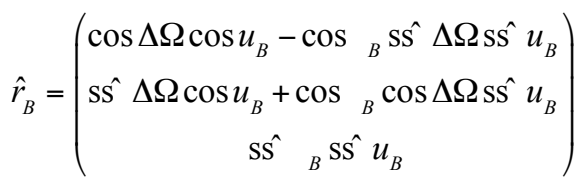

where $\hat{r}_{i}$ is a unit vector, $\quad={ }_{B} \quad{ }_{A}$ and $u_{i}=f_{i}+{ }_{i}$ from which

$\cos S_{A B}=\cos \Delta \Omega \cos u_{A} \cos u_{B}-\cos { }_{B} \sin \Delta \Omega \cos u_{A} \sin u_{B}+$ $\cos { }_{A} \sin \Delta \Omega \sin u_{A} \cos u_{B}+\cos { }_{A} \cos { }_{B} \cos \Delta \Omega \sin u_{A} \sin u_{B}$ $+\sin { }_{A} \sin { }_{B} \sin u_{A} \sin u_{B}$

Adopting reference frame is an equatorial system with the positive $\mathrm{X}$-axis toward the vernal equinox, $\mathrm{Z}$ - axis toward the north pole of the equator, and the $\mathrm{Y}$-axis completing a right handed system. We note in the development of $\cos S_{12}$ and $\cos S_{\mathrm{cs}}$ the appearance of $I_{1}, I_{2}$ and $I_{3}$ the inclinations, to the equator, of the orbits of the satellite, the moon and the sun respectively. The final expressions for $\cos S_{12}$ and $\cos S_{13}$ become after some lengthy algebra:

$\cos s_{12}=\frac{1}{2}\left[\left(1-c_{1} c_{2}\right) \cos \Delta \Omega_{2} \cos \left(u_{1}+u_{2}\right)+\right.$

$\left(1+c_{1} c_{2}\right) \cos \Delta \Omega_{2} \cos \left(u_{1}-u_{2}\right)$

$\left.+\left(c_{1}-c_{2}\right) \sin \Delta \Omega_{2} \sin \left(u_{1}+u_{2}\right)+\left(c_{1}+c_{2}\right) \sin \Delta \Omega_{2} \sin \left(u_{1}-u_{2}\right)\right]$

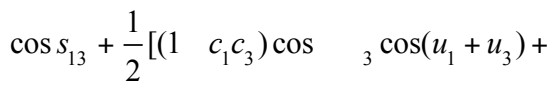

$\left(1+c_{1} c_{3}\right) \cos \Delta \Omega_{3} \cos \left(u_{1}-u_{3}\right)$

$\left.+\left(c_{1}-c_{3}\right) \sin \Delta \Omega_{3} \sin \left(u_{1}+u_{3}\right)+\left(c_{1}+c_{3}\right) \sin \Delta \Omega_{3} \sin \left(u_{1}-u_{3}\right)\right]$

where $c_{i}=\cos I_{i}, s_{i}=\sin I_{i}$ ann $\Delta \Omega_{j}=\Omega_{j}-\Omega_{i}$.

Substituting Eqs. (8) into Eq. (7) and dropping the subscript ' 1 ' designating the satellite, we obtain the force function $U$ grav.

\subsection{The Force Functions Due to the Oblateness}

We have so far considered the earth as a point mass introducing the effect of earth oblateness we can write the disturbing function in the form:

$U=\frac{\mu}{r_{1}}+U_{g a r v .}+U_{o b}$

where $U_{o b}$ represents the contribution of the oblateness of the earth to the disturbing function. Assuming an axially symmetric geopotential truncated beyond the fourth zonal harmonic, the disturbing function due to earth oblateness acquires the form (e. g. Fitzpatrick (1970) [24])

$U_{o o .}=\frac{\mu}{r}_{r=2}^{4} J_{r}\left(\frac{R}{r}\right)^{r} p_{r}(\sin )$

where $r$ and $\delta$ are the geocentric distance end declination of the satellite, $J_{n}$ are harmonic coefficients with $J_{2} \quad 10^{3}$ and $J_{n}\left(n>() \approx 0^{-6}, R\right.$ is the equatorial radius of the earth, $p_{n} \sin$ ( are Legendre polynomials.

\subsection{The Force Functions Due to Solar Radiation Pressure}

Consider $\sigma$ to be the absolute value of the acceleration of the satellite arising from the solar radiation pressure. Then the disturbing function for the radiation pressure will be given by (see the earlier section, 'the Angles $S_{13}$ and $S_{13}^{\prime}$ ).

$R=r \cos S_{13}^{\prime}$

The order of magnitude of the radiation pressure acceleration depends on the area to the mass ratio of the satellite. If the area to the mass ratio of the satellite is of $\mathrm{O}\left(10^{1}\right)$ then the disturbing acceleration due to the radiation pressure is of fourth order.

\subsection{The Force Functions in Terms of the Delaunay Ele- ments}

Reproducing Eq. (9), the force function can now be written as:

$$
U=\frac{\mu}{r}+U_{\text {grav. }}+U_{o b .}+R_{\sigma}
$$

where $U_{\text {grrv }}, U_{o b}$. and $R_{\sigma}$ are given by Eq.(7), Eq.(10) and Eq.(11) respectively.

We can assume, with sufficient accuracy, that the moon and the sun move in circular orbits so that $u_{2}=n_{2} t+$ constant and $u_{3}=n_{3} t+$ constant

where $n_{2}$ and $n_{3}$ are the respective mean motions. In what follows we form the Hamiltonian of the problem in terms of the Delaunay elements. We have

$$
F^{\prime} \mu \frac{\mu^{2}}{2 L^{2}}+U_{\text {grav. }}+U_{o F}+R_{\sigma}
$$

Before proceeding to write $U_{\text {grav. }}, U_{o b \text {. }}$ and $R$ in terms of Delaunay's elements we note that the time $t$ appears explicitly in $F^{\prime}$ through its explicit appearance in $u_{2}, u_{3}$ and $R_{\sigma}$. Hence we augment the set by the pair $k_{\circ}=n_{2} t+$ constant,$k_{\Theta}=n_{3} t+$ constant, and their conjugates $K$ and $K$ Hori (1966) [25]

Our set then consists of the ten elements

$$
l=\text { mean anomaly, } \quad L=(\mu a)^{/ /}
$$


$g=$ argument of perigee, $G=L\left(1-e^{2}\right)^{1 / 2}$

$h=\Omega-\Omega$

$H=G c$

$=$ longitude of the node measured, from the node of the moon's orbit

$\begin{array}{ll}k_{o}=u_{2}, & K_{\circ} \\ k_{\Theta}=u_{3}, & K_{\Theta}\end{array}$

In terms of this set the Hamiltonian becomes

$F=\frac{\mu^{2}}{2 L^{2}}-n_{2} k_{\circ}-n_{3} k_{\Theta}+U_{\text {grav. }}+U_{o b .}+R_{\sigma}$

\section{THE HAMILTONIAN}

Regarding $n_{2}$ (the mean motion of the moon) as the small parameter of the problem, therefore the perturbing terms in the Hamiltonian can be grouped and expressed in expandable form as:

$$
F=\sum_{n=0}^{5} \frac{\varepsilon^{n}}{n !} F_{n} \quad\left(\varepsilon=n_{2}\right)
$$

with

$$
\begin{aligned}
F_{o} & =\frac{\mu^{2}}{2 L^{2}} \\
F_{1} & =-K_{o} \\
F_{2} & =\gamma_{2} \phi^{3} z_{2}+A K_{\Theta} \\
F_{3} & =0 \\
F_{4} & =\sum_{m=1}^{5} F_{4 m} \\
F_{41}=A_{1} \frac{L^{4} x_{1}}{\phi^{2}}, & F_{42}=A_{3} \frac{L^{4} x_{3}}{\phi^{2}}, F_{43}=\gamma_{3} \phi^{4} z_{3}, F_{44}=\gamma_{4} \phi^{5} z_{4}, F_{45}=R_{\sigma} . \\
F_{5} & =A_{2} \frac{L^{6} x_{2}}{\phi^{3}}
\end{aligned}
$$

where

$x_{1}=\theta_{\circ}+\theta_{1} \cos h+\left(\theta_{2}-\theta_{1} \cos h+\theta_{3} \cos 2 h\right) \cos 2 u$

$+\left(\theta_{3}-\theta_{1} \cos h+\theta_{2} \cos 2 h\right) \cos 2 k_{\circ}-\left(\theta_{4} \sin 2 h+\theta_{5} \sin h\right) \sin 2 u$

$-\left(\theta_{6} \sin h+\theta_{7} \sin 2 h\right) \sin 2 k_{\circ}+\theta_{8} \cos 2 h \cos 2\left(u+k_{\circ}\right)$

$+\left[\theta_{9} \cos 2 h+\left(4 \theta_{10}+\theta_{1}\right) \cos h\right] \cos \left(u-k_{\mathrm{o}}\right)+2 \theta_{10} \sin h \sin 2\left(u+k_{\mathrm{o}}\right)$

$-\left[\left(\theta_{6}-\theta_{5}\right) \sin h+\theta_{11} \sin 2 h\right] \sin 2\left(u-k_{\circ}\right)$

$x_{2}=\theta_{12} \cos h \cos \left(u+k_{\mathrm{o}}\right)+\theta_{14} \cos h \cos \left(u-k_{\mathrm{o}}\right)+\theta_{16} \cos 3 h \cos 3\left(u-k_{\mathrm{o}}\right)$

$+\left(\theta_{17} \cos h+\theta_{18} \cos 3 h\right)\left[\cos \left(3 u-k_{\circ}\right)+\cos \left(u-3 k_{\circ}\right)\right]$

$-\theta_{20} \sin 3 h \sin 3\left(u-k_{\mathrm{o}}\right)-\theta_{21} \sin h \sin \left(u+k_{\mathrm{o}}\right)-\theta_{22} \sin h \sin \left(u-k_{\mathrm{o}}\right)$

$-\left(\theta_{23} \sin h+\theta_{18} \sin 3 h\right)\left[\sin \left(3 u-k_{\mathrm{o}}\right)-\sin \left(u-3 k_{\mathrm{o}}\right)\right]$

$x_{3}=\theta_{24}+\theta_{25} \cos 2(h+\Gamma) \cos 2 u+\theta_{25} \cos 2 k_{\Theta}-\theta_{26} \sin 2(h+\Gamma) \sin 2 u$

$+\theta_{27} \cos 2(h+\Gamma) \cos 2\left(u+k_{\Theta}\right)+\theta_{28} \cos 2(h+\Gamma) \cos 2\left(u-k_{\Theta}\right)$

$-\theta_{29} \sin 2(h+\Gamma) \sin 2\left(u-k_{\Theta}\right)$

$z_{2}=\frac{1}{L^{6}}\left(\theta_{30}+\frac{3}{2} \theta_{2} \cos 2 u\right)$,

$z_{3}=\frac{1}{L^{8}}\left(\theta_{31} \sin u+\theta_{32} \sin 3 u\right)$,

$z_{4}=\frac{1}{L^{10}}\left(\theta_{33}+\theta_{34} \cos 2 u+\theta_{35} \cos 4 u\right)$
$R_{\sigma}=-\frac{1}{2} \sigma r\left[\left(1-c c_{3}\right) \cos (h+\Gamma) \cos \left(u+k_{\Theta}\right)+\right.$

$\left(1+c c_{3}\right) \cos (h+\Gamma) \cos \left(u-k_{\Theta}\right)-\left(c-c_{3}\right) \sin (h+\Gamma) \sin \left(u+k_{\Theta}\right)$

$\left.-\left(c+c_{3}\right) \sin (h+\Gamma) \sin \left(u-k_{\Theta}\right)\right]$

$=-\frac{1}{4} \sigma r \sum_{i=-1}^{1} \sum_{j=-1}^{1} \vartheta_{i j}\left\{\cos \left[g+i(h+\Gamma)+j k_{\Theta}\right] \cos f\right.$

$\left.-\sin \left[g+i(h+\Gamma)+j k_{\Theta}\right] \sin f\right\}$

the A's and $\gamma$ 's are zero order quantities defined by:

$A=-2 n_{3} / \varepsilon^{2} \quad A_{1}=\frac{9}{2 \varepsilon^{4}} \frac{k^{2} m_{2}}{\mu^{2} a_{2}^{3}}, \quad A_{2}=\frac{75}{32 \varepsilon^{5}} \frac{k^{2} m_{2}}{\mu^{3} a_{2}^{4}}$,

$A_{3}=\frac{9}{2 \varepsilon^{4}} \frac{k^{2} m_{3}}{\mu^{2} a_{3}^{3}}$

$\gamma_{2}=\frac{1}{2 \varepsilon^{2}} R^{2} \mu^{4} J_{2}, \quad \gamma_{3}=\frac{3}{\varepsilon^{4}} R^{3} \mu^{5} J_{3}, \quad \gamma_{4}=-\frac{3}{8 \varepsilon^{4}} R^{4} \mu^{6} J_{4}$,

$\Gamma=\Omega_{2}-\Omega_{3}$,

and $\theta_{i}(i=0,1,2, \ldots . ., 30)$ are functions of $c_{i}$ and $s_{i}$, given by:

$$
\begin{aligned}
& \theta_{0}=(4 / 3)-2\left(s^{2}+s_{2}^{2}\right), \quad \theta_{1}=4 s s_{2} c c_{2}, \quad \theta_{2}=2 s^{2}, \\
& \theta_{3}=2 s_{2}^{2}, \quad \theta_{4}=c s_{2}^{2}, \quad \theta_{5}=-2 s s_{2} c_{2}, \\
& \theta_{6}=2 s s_{2} c, \quad \theta_{7}=-c_{2} s^{2}, \quad \theta_{8}=2-s^{2}-s_{2}^{2}-2 c c_{2}, \\
& \theta_{9}=2-s^{2}-s_{2}^{2}+2 c c_{2}, \\
& \theta_{10}=s s_{2}, \quad \theta_{11}=c+c c_{2}^{2}+c^{2} c_{2}+c_{2} \\
& \theta_{12}=-(102 / 5)+(347 / 5) c c_{2}-6 c c_{2}^{3}+6 c^{2}- \\
& 30 c_{2}^{2} c^{2}-6 c^{3} c_{2}-18 c^{3} c_{2}^{3}+6 c_{2}^{2}, \\
& \theta_{13}=1-c^{2}+c^{2} c_{2}^{2}+c_{2}^{2}, \\
& \theta_{14}=-(147 / 5)-(27 / 5) c c_{2}+3 c c_{2}^{3}+3 c^{2}+33 c^{2} c_{2}^{2}+3 c^{3} c_{2}+9 c^{3} c_{2}^{3}+3 c_{2}^{2}, \\
& \theta_{15}=3\left(1+c c_{2}-c c_{2}^{3}-c^{2}+c^{2} c_{2}^{2}-c^{3} c_{2}-c^{3} c_{2}^{3}-c_{2}^{2}\right), \\
& \theta_{18}=1+4 c c_{2}+c^{2}+c^{2} c_{2}^{2}+c_{2}^{2}, \\
& \theta_{19}=3\left(c-c c_{2}^{2}-c^{2} c_{2}+c^{2} c_{2}^{3}-c^{3}+c^{3} c_{2}^{2}+c_{2}-c_{2}^{3}\right), \\
& \theta_{17}=3\left(3+c c_{2}+c c_{2}^{3}-c^{2}-c^{2} c_{2}^{2}+c^{3} c_{2}-3 c^{3} c_{2}^{3}-c_{2}^{2}\right), \\
& \theta^{2}+c^{2} c_{2}^{2}+3 c_{2}^{2},
\end{aligned}
$$


$\theta_{21}=-(162 / 5) c-30 c c_{2}^{2}+30 c^{2} c_{2}-6 c^{2} c_{2}^{3}+18 c^{3}+6 c^{3} c_{2}^{2}+(222 / 5) c_{2}-18 c_{2}^{3}$,

$\theta_{22}=-(177 / 5) c+33 c c_{2}^{2}+33 c^{2} c_{2}+3 c^{2} c_{2}^{3}+9 c^{3}+3 c^{3} c_{2}^{2}-(177 / 5) c_{2}+9 c_{2}^{3}$,

$\theta_{23}=3\left(c+c c_{2}^{2}-c^{2} c_{2}-c^{2} c_{2}^{3}-3 c^{3}+c^{3} c_{2}^{2}-c_{2}+3 c_{2}^{3}\right)$,

$\theta_{24}=(4 / 3)-2 s_{3}^{2}, \quad \theta_{25}=2 s_{3}^{2}$,

$\theta_{26}=c s_{3}^{2}, \quad \theta_{27}=2-s_{3}^{2}-2 c c_{3}, \theta_{28}=2-s_{3}^{2}+2 c c_{3}$,

$\theta_{29}=c+c c_{3}^{2}+c^{2} c_{3}+c_{3}, \theta_{30}=2-3 s^{2}$,

$\vartheta_{-1,-1}=1-c-c_{3}+c c_{3} \quad \vartheta_{1,-1}=1+c+c_{3}+c c_{3}$

$\vartheta_{-1,1}=1-c+c_{3}-c c_{3}$ and $\vartheta_{1,1}=1+c-c_{3}-c c_{3}$.

\section{THE PROCEDURE FOR SOLUTION}

The Hamiltonian $F$ of the problem defined by Equations (15) and (16) can be represented by $F=F_{0}+\sum_{n=1}^{5} \frac{\varepsilon^{n}}{n !} F_{n}$

where $F_{0}$ is the unperturbed Hamiltonian. It represents the integrable part of the problem. Then the equations of motion can be written in the form

$\dot{u}=\frac{\delta F}{\delta U}, \quad \dot{U}=-\frac{\delta F}{\delta u}$

where $(u, U)$ is the ten-vector of adopted canonical variables $\left(l, g, h, k_{o}, k_{\Theta} ; L, G, H, K_{o}, K_{\Theta}\right)$.

To develop the solution including the effects of the perturbing Hamiltonian $F_{n}$, it is required now to construct three canonical transformations $(u, U ; \varepsilon) \rightarrow\left(u^{\prime}, U^{\prime}\right)$ and $\left(u^{\prime}, U^{\prime}\right.$; $\varepsilon) \rightarrow\left(u^{\prime \prime}, U^{\prime \prime}\right)$ and $\left(u^{\prime \prime}, U^{\prime \prime} ; \varepsilon\right) \rightarrow\left(u^{\prime \prime \prime}, U^{\prime \prime \prime}\right)$ analytic in $\varepsilon$ at $\varepsilon=0$, to remove in succession the fast and slow angles from $F_{n}$. This process reduces $U^{\prime \prime \prime}$ to constants and $u^{\prime \prime \prime}$ to linear functions of time.

A theorem by Lie has been applied to construct explicit transformations. Deprit (1969) [22] constructed an algorithm to generate the new Hamiltonian recursively using the Lie transform, while Kamel (1969) [23] simplified Deprit's algorithm. We now outline the perturbation technique based on Kamel [23] to evaluate the secular and the periodic perturbations.

The transformed Hamiltonians and the corresponding generators will be assumed expandable as

$$
\begin{aligned}
& F^{*}\left(-, u_{2}^{\prime}, u_{3}^{\prime}, u_{4}^{\prime}, u_{5}^{\prime} ; U^{\prime} ; \varepsilon\right)=F_{o}^{*}\left(U_{1}^{\prime}\right)+ \\
& \sum_{n=1}^{6} \frac{\varepsilon^{n}}{n !} F_{n}^{*}\left(-, u_{2}^{\prime}, u_{3}^{\prime}, u_{4}^{\prime}, u_{5}^{\prime} ; U^{\prime}\right) \\
& w\left(u^{\prime}, U^{\prime} ; \varepsilon\right)=\sum_{n=0}^{4} \frac{\varepsilon^{n}}{n !} w_{n+1}\left(u^{\prime} ; U^{\prime}\right) \\
& F^{* *}\left(-,-, u_{3}^{\prime \prime}, u_{4}^{\prime \prime}, u^{\prime \prime}{ }_{5} ; U^{\prime \prime} ; \varepsilon\right)=F_{o}^{* *}\left(U_{1}^{\prime \prime}\right)+F_{1}^{* *}\left(U_{2}^{\prime \prime}\right)+ \\
& \sum_{n=2}^{6} \frac{\varepsilon^{n}}{n !} F_{n}^{* *}\left(-,-, u^{\prime \prime}{ }_{3}, u^{\prime \prime}{ }_{4}, u^{\prime \prime}{ }_{5} ; U^{\prime \prime}\right)
\end{aligned}
$$

$$
\begin{aligned}
& w^{*}\left(-, u_{2}^{\prime \prime}, u_{3}^{\prime \prime}, u_{4}^{\prime \prime}, u_{5}^{\prime \prime} ; U^{\prime \prime} ; \varepsilon\right)=\sum_{n=0}^{3} \frac{\varepsilon^{n}}{n !} w_{n+1}^{*}\left(-, u_{2}^{\prime \prime}, u_{3}^{\prime \prime}, u_{4}^{\prime \prime}, u_{5}^{\prime \prime} ; U^{\prime \prime}\right) \\
& F^{* * *}\left(-; U^{\prime \prime \prime} ; \varepsilon\right)=F_{o}^{* * *}\left(U_{1}^{\prime \prime \prime}\right)+F_{1}^{* * *}\left(U_{2}^{\prime \prime \prime}\right)+\sum_{n=2}^{6} \frac{\varepsilon^{n}}{n !} F_{n}^{* * *}\left(-, U^{\prime \prime \prime}\right) \\
& w^{* *}\left(-,-, u_{3}^{\prime \prime \prime}, u_{4}^{\prime \prime \prime}, u_{5}^{\prime \prime \prime} ; U^{\prime \prime \prime} ; \varepsilon\right)=\sum_{n=0}^{3} \frac{\varepsilon^{n}}{n !} w_{n+1}^{* *}\left(-,-, u_{3}^{\prime \prime \prime}, u_{4}^{\prime \prime \prime}, u_{5}^{\prime \prime \prime} ; U^{\prime \prime \prime}\right)
\end{aligned}
$$

\section{THE NORMALIZED HAMILTONIAN}

As is clear from the process we have five angles of different rates of change. These angles are: the mean anomaly $l$ is the fast variable, the Moon's mean longitude $k_{o}$ is the intermediate variable, while the argument of perigee $g$, the longitude of the ascending node $h$ and the Sun's mean longitude $k_{\Theta}$ are the slow variables. We thus need three canonical transformations to eliminate in succession the short, the intermediate and the long period terms from the Hamiltonian using a perturbation technique based on Lie series and Lie transform Kamel [23].

\subsection{Elimination of the Short Period Terms}

Since the integrable part of the Hamiltonian $F_{o}$ depends on $L$, then the mean anomaly $l$ is considered as the fast variables of the problem. We thus use the Lie transform to eliminate the short periodic terms from the Hamiltonian with the averages being taken over $l$. This step effected by a canonical transformation from the old Hamiltonian Eqns. (15) to a new one $F^{*}\left(-, g^{\prime}, h^{\prime}, k_{o}^{\prime}, k_{\Theta}{ }^{\prime} ; L^{\prime}, G^{\prime}, H^{\prime}, K_{o}{ }^{\prime}, K_{\Theta}{ }^{\prime}\right)$ that is to be performed via a generating function $W\left(l^{\prime}, g^{\prime}, h^{\prime}, k_{o}{ }^{\prime}, k_{\Theta}{ }^{\prime} ; L^{\prime}, G^{\prime}, H^{\prime}, K_{o}{ }^{\prime}, K_{\Theta}{ }^{\prime}\right)$.

\subsubsection{Results of the Computations}

Applying the recursive process developed by Deprit [22] and Kamel [23] to the Hamiltonian defined by Eqs. (15) and (16) the following results for $F^{*}$ and $W$ are obtained at different orders after some lengthy manipulations. All variables are understood to be single - primed, but the primes are dropped for the sake of simplicity of writing.

$$
\begin{aligned}
F_{\circ}^{*} & =\frac{\mu^{2}}{2 L^{2}} \\
F_{1}^{*} & =-K_{\circ} \\
F_{2}^{*} & =\gamma_{2} \theta_{30} \eta_{33}+A K_{\Theta},\left(\eta_{i j}=L^{-i} G^{-j}, \eta=L / G\right) \\
F_{3}^{*} & =0 \\
F_{4}^{*}= & L^{4}\left[a_{0}\left(1+\frac{3}{2} e^{2}\right)+\frac{5}{2} e^{2}\left(a_{2} \cos 2 g+b_{2} \sin 2 g\right)\right]+\sum_{i=0}^{1}\left(\chi_{i}^{c} \cos 2 i g\right. \\
& \left.+\chi_{i}^{s} \sin i g\right)+\frac{3 \sigma a e}{8} \sum_{i=-1}^{1} \sum_{j=-1}^{1} \vartheta_{i j} \cos \left(g+i(h+\Gamma)+j k_{\Theta}\right) \\
F_{5}^{*}= & A_{2} L^{6}\left[\left(-\frac{5}{2} e-\frac{15}{8} e^{3}\right)\left(a_{1} \cos g+b_{1} \sin g\right)-\frac{35}{8} e^{3}\left(a_{3} \cos 3 g\right.\right. \\
& \left.\left.+b_{3} \sin 3 g\right)\right]-\frac{25}{2} \frac{A_{1}}{\mu^{2}}\left(\eta_{-6,-1}-\eta_{-4,-3}\right)\left[\left(\tilde{v}_{3}^{s} \cos 2 g\right.\right. \\
& \left.\left.-\tilde{v}_{2}^{s} \sin 2 g\right) \sin 2 k_{o}+\left(\tilde{v}_{3}^{c} \cos 2 g-\tilde{v}_{2}^{c} \sin 2 g\right) \cos 2 k_{o}\right]
\end{aligned}
$$




$$
\begin{aligned}
& F_{6}^{*}=\sum_{i=1}^{6} F_{6 i}^{*} \\
& F_{61}^{*}=-15 \sum_{j=0}^{2} d_{j} \cos 2 j g \\
& F_{62}^{*}=\sum_{j=1}^{3}\left(X_{j}^{c} \cos j k_{o}+X_{j}^{s} \sin j k_{\mathrm{o}}\right) \\
& F_{63}^{*}=10 \frac{\gamma_{2}}{\mu^{2}} \sum_{j=0}^{1}\left(M_{j}^{c} \cos 2 j k_{o}+M_{j}^{s} \sin 2 j k_{\mathrm{o}}\right) \\
& F_{64}^{*}=-5 \gamma_{2} \sum_{j=0}^{1}\left(N_{j}^{c} \cos 2 j k_{o}+N_{j}^{s} \sin 2 j k_{\mathrm{o}}\right) \\
& F_{65}^{*}=5 \sum_{j=0}^{1}\left(m_{j}^{c} \cos 2 j k_{o}+m_{j}^{s} \sin 2 j k_{\mathrm{o}}\right) \\
& F_{66}^{*}=10 \sum_{j=0}^{1}\left(n_{j}^{c} \cos 2 j k_{o}+n_{j}^{s} \sin 2 j k_{\mathrm{o}}\right)
\end{aligned}
$$

$W_{5}=W_{51}+W_{52}$

with

$W_{51}=-\frac{A_{2}}{\mu^{2}} L^{9}\left\{\sum_{i=0}^{4} \sum_{j=-3}^{3}\left[\xi^{s_{i j}} \sin (i E+j g)+\xi^{\varepsilon_{i j}} \cos (i E+j g)\right]+\xi(E-l)\right\}$

$W_{52}=\frac{5 L^{10}}{\mu^{2}}\left\{\sum_{i=1}^{4} \sum_{j=-1}^{1}\left[\xi_{i j}^{c} \cos (i E+2 j g)+\xi_{i j}^{s} \sin (i E+2 j g)\right]+\xi_{o 0}(E-l)\right\}$ where $\quad F_{i j}=i f+i g$

\subsection{Intermediate - Period Transformation}

In this section the intermediate - period terms (those periodic in $k_{\mathrm{o}}$ ) are eliminated via a second canonical transformation, leaving the Hamiltonian containing only the slow angle variables. The procedure is essentially similar to the short periodic terms but with averages being taken over $k_{o}$

\subsubsection{Results of Computation}

Application of the above procedure to the Hamiltonian $F^{*}$ given by (21) to (27) yields: (all variables on the right are now double primed)

Where the quantities $a_{i}, b_{i}, \chi_{i}^{c}, \chi_{i}^{s}, \tilde{v}_{i}^{c}, \tilde{v}_{j}^{s}, X_{j}^{c}, X_{j}^{s}, d_{j}, M_{j}^{c}, M_{j}^{s}, N_{j}^{c}, N_{j}^{s}, m_{j}^{s}, m_{j}^{c}, n_{j}^{c}$ and $n_{j}^{s}$ are functions of the action and angular variables.

$W_{1}=0$

$W_{2}=\frac{-\gamma_{2}}{\mu^{2} G^{3}}\left\{\theta_{30}(f-l+e \sin f)+\right.$

$\left.\frac{3}{2} \theta_{2}\left[\frac{e}{2} \sin F_{12}+\frac{1}{2} \sin F_{22}+\frac{e}{6} \sin F_{32}\right]\right\}$

$$
\begin{aligned}
& W_{3}=0 \\
& W_{4}=\sum_{i=1}^{4} W_{4 i}
\end{aligned}
$$

with

$$
\begin{aligned}
W_{41}= & -\frac{L^{7}}{\mu^{2}}\left\{\sum_{i=1}^{3} \sum_{j=-1}^{1}\left[B_{i j}^{c} \cos (i E+2 j g)+B_{i j}^{s} \sin (i E+2 j g)\right]+B_{\circ \circ}(E-l)\right\} \\
W_{42}= & -\frac{L^{3}}{\mu^{2}}\left\{\sum_{i=1}^{7} \sum_{j=-1}^{3}\left[D_{i j}^{c} \cos F_{i j}+D_{i j}^{s} \sin F_{i, 2 j}\right]+D_{\circ \circ}(f-l)\right\} \\
W_{43}= & -\frac{9 \gamma_{2}^{2}}{\mu^{4}} \eta_{52}\left\{\sum _ { i = 1 } ^ { 6 } \sum _ { j = - 1 } ^ { 2 } \left[\psi_{i j} \sin F_{i, 2 j}+\sum_{j=0}^{1} \tilde{\psi}_{j}(f-l) \cos 2 j g\right.\right. \\
& \left.+\sum_{i=1}^{4} \psi_{i}+\frac{1}{e^{2}}\left(5 s^{4}-4 s^{2}\right)\left(-\eta^{5}+3 \eta^{3}-2 \eta^{2}\right)(E-l) \cos 2 g\right\} \\
W_{44}= & \frac{L^{3} \sigma a}{16 \mu^{2}} \sum_{i=-1}^{1} \sum_{j=-1}^{1} \vartheta_{i j}\left\{\left[4\left(1+e^{2}\right) \sin \mathrm{E}-e \sin 2 \mathrm{E}-6 e(\mathrm{E}-l)\right] \times\right. \\
& \cos \left(g+i(h+\Gamma)+j k_{\Theta}\right)+\frac{1}{\eta}(4 \cos \mathrm{E}-e \cos 2 \mathrm{E}) \times \\
& \left.\sin \left(g+i(h+\Gamma)+j k_{\Theta}\right)\right\}
\end{aligned}
$$

$$
\begin{aligned}
& F_{0}^{* *}=\frac{\mu^{2}}{2 L^{2}} \\
& F_{1}^{* *}=F_{1}^{*}=-K_{\circ}, F_{2}^{* *}=F_{2}^{*}=\gamma_{2} \theta_{30} \eta_{33}+A K_{\circ}, F_{3}^{* *}=0 \\
& F_{4}^{* *}=L^{4}\left[\left(1+\frac{3}{2} e^{2}\right) \lambda_{36}+\frac{5}{2} e^{2}\left(\lambda_{39} \cos 2 g+\lambda_{42} \sin 2 g\right)\right] \\
& +\sum_{i=0}^{1}\left(x_{i}^{c} \cos 2 i g+x_{i}^{s} \sin i g\right)+\frac{3 \sigma a e}{8} \sum_{i=-1}^{1} \sum_{j=-1}^{1} \vartheta_{i j} \cos \left(g+i(h+\Gamma)+j k_{\Theta}\right) \\
& F_{5}^{* *}=0 \\
& F_{6}^{* *}=-15 \sum_{i=0}^{2} d_{i} \cos 2 i g+\frac{10 \gamma_{2}}{\mu^{2}} M_{0}^{c}+5 m_{0}^{c}+10 n_{0}^{c}-5 \gamma_{2} N_{\circ}^{c} \\
& W_{1}^{*}=W_{2}^{*}=0 \\
& W_{3}^{*}=\frac{L^{4}}{8}\left\{\left[\left(1+\frac{3}{2} e^{2}\right) \lambda_{37}+\frac{5}{2} e^{2}\left(\lambda_{40} \cos 2 g+\lambda_{43} \sin 2 g\right)\right] \cos 2 k_{\circ}\right. \\
& \left.\quad-\left[\left(1+\frac{3}{2} e^{2}\right) \lambda_{38}+\frac{5}{2} e^{2}\left(\lambda_{41} \cos 2 g+\lambda_{44} \sin 2 g\right)\right] \sin 2 k_{\circ}\right\} \\
& W_{4}^{* *}=-\frac{1}{5} \sum_{i=1}^{3} \frac{1}{i}\left(v_{i}^{c} \sin i k_{\circ}-v_{i}^{s} \cos i k_{\circ}\right)
\end{aligned}
$$

\subsection{Elimination of Long- Period Terms}

The long-period terms, those periodic in $g, h$ and $k_{\Theta}$ will be eliminated and the elements of long period transformation will be obtained. The transformation is being made via a generator $W^{* *}$ where the old and new Hamiltonians are related through

$$
\begin{aligned}
& F^{* * *}\left(-, g^{\prime \prime}, h^{\prime \prime},-, k_{\Theta}^{\prime \prime} ; L^{\prime \prime}, G^{\prime \prime}, H^{\prime \prime}, K_{o}^{\prime \prime}, K_{\Theta}^{\prime \prime}\right)= \\
& F^{* * * *}\left(L^{\prime \prime \prime}, G^{\prime \prime \prime}, H^{\prime \prime \prime}, K_{o}^{\prime \prime \prime}, K_{\Theta}^{\prime \prime \prime}\right)
\end{aligned}
$$

The transformation equations are essentially the same as those of last section with relevant changes of primes and asterisks and the averages being taken over $g, h$ and $k_{\Theta}$. 


\subsubsection{Results of Computation}

All variables on the right are now triple primed

$F_{0}^{* * * *}=\frac{\mu^{2}}{2 L^{2}}$

$F_{1}^{* * * *}=-k_{o}$

$F_{2}^{* * * *}=F_{2}^{* * *}=\gamma_{2} \theta_{30} \eta_{33}+A K_{\Theta}$

$F_{3}^{* * *}=0$

$F_{4}^{* * * *}=L^{4}\left[\left(1+\frac{3}{2} e^{2}\right)\left(A_{1} \theta_{0}+A_{3} \theta_{24}\right)\right.$

$F_{5}^{* * * *}=0$

$$
\begin{aligned}
F_{6}^{*+4+s}= & -15 d_{0}+\frac{\gamma_{2}}{\mu^{2}}\left\{10\left(A_{1} L^{4} \tilde{\sigma}_{00}+\tilde{\chi}_{0}^{c}\right)+5\left[\gamma_{4} \tilde{M}_{0}^{c}+L^{4}\left(A_{1} \tilde{p}_{00}^{c}+A_{3} \tilde{\tilde{p}}_{000}^{c}\right)\right]\right\} \\
& +10\left[\tilde{W}_{z 00}^{c}+\tilde{W}_{z 2,-1}^{c}+\tilde{W}_{z,-21}^{c}+A_{1}\left(\tilde{\lambda}_{z 000}^{c}+\tilde{\lambda}_{z 01,-1}^{c}+\tilde{\lambda}_{z 0,-11}^{c}\right)+A_{3} \tilde{\sigma}_{z 00}^{c}\right] \\
& +\frac{1}{2} \sum_{i=1}^{4}\left[\bar{J}_{0}^{i}+\bar{\rho}_{0}^{i}+A_{1}\left(\bar{J}_{1}^{i}+\bar{\rho}_{1}^{i}\right)+A_{1} A_{3}\left(\bar{J}_{13}^{i}+\bar{\rho}_{13}^{i}\right)+A_{1}^{2}\left(\bar{J}_{11}^{i}+\bar{\rho}_{11}^{i}\right)\right. \\
& \left.+A_{3}\left(\bar{J}_{3}^{i}+\bar{\rho}_{3}^{i}\right)+A_{3}^{2}\left(\bar{J}_{33}^{i}+\bar{\rho}_{33}^{i}\right)\right]
\end{aligned}
$$

$$
\begin{aligned}
& W_{4}^{* *}=-\sum_{i=1}^{2} \frac{d_{i}}{2 i \tilde{\alpha}_{3}} \sin 2 i g+\frac{2 \gamma_{2}}{3 \mu^{2}}\left\{L ^ { 4 } \left[A_{1} \sum_{i=0}^{2^{\prime}} \sum_{j=-2}^{2^{\prime}} \frac{\tilde{\sigma}_{i j}}{i \tilde{\alpha}_{1}+2 j \tilde{\alpha}_{3}} \sin (i h+2 j g)\right.\right. \\
& +A_{3} \sum_{m=0}^{2} \sum_{i=-1}^{1} \sum_{j=-2}^{2} \frac{\tilde{\tilde{\sigma}}_{m i j}}{2\left(m \tilde{\alpha}_{1}+i \tilde{\alpha}_{2}+j \tilde{\alpha}_{3}\right)} \sin 2[m(h+\Gamma) \\
& \left.\left.+i k_{\Theta}+j g\right]+\sum_{i=0}^{2} \frac{1}{\tilde{\alpha}_{3}}\left(\frac{1}{2 i} \tilde{\chi}_{i}^{c} \sin 2 i g-\frac{1}{1+i} \tilde{\chi}_{i}^{s} \cos (i+1) g\right)\right\} \\
& -\frac{\gamma_{2}}{3 \mu^{2}}\left\{A_{1} \sum_{i=0}^{2} \sum_{j=-1}^{1} \frac{\tilde{N}_{i j}}{i \tilde{\alpha}_{1}+2 j \tilde{\alpha}_{3}} \sin (2 j g+i h)\right. \\
& +A_{3} \sum_{i=-1}^{1^{\prime}} \sum_{j=-1}^{1^{\prime}} \frac{\tilde{\tilde{N}}_{i j}}{2\left(\tilde{\alpha}_{1}+i \tilde{\alpha}_{2}+j \tilde{\alpha}_{3}\right)} \sin 2\left(j g+h+\Gamma+i k_{\Theta}\right)-27 \frac{\gamma_{2}^{2}}{\mu^{2}}\left(1-5 C^{2}\right) \times \\
& \eta_{86}\left(\sum_{i=1}^{6 z} \sum_{j=-1}^{2^{\prime}} \frac{j}{i \tilde{\alpha}_{3}} \psi_{i j} \tilde{g}_{01 i}^{c} \sin 2 i g+\frac{1}{2 \tilde{\alpha}_{3}} \psi \sin 2 g\right)-3\left(1-5 C^{2}\right) \eta_{04} \sum_{i=-1}^{3^{\prime}} \sum_{j=1}^{7^{\prime}} \frac{1}{\tilde{\alpha}_{3}} \times \\
& \left.\left(D_{i j}^{s} \sin 2 j g+D_{i j}^{c} \cos j g\right) \tilde{g}_{01 i}^{c}\right\}+\frac{\gamma_{2}}{3 \mu^{2}}\left\{\sum _ { i = 0 } ^ { 3 ^ { \prime } } \left(\gamma_{4} \tilde{M}_{i}^{c} \frac{1}{2 i \tilde{\alpha}_{3}} \sin 2 i g-\gamma_{3} \tilde{M}_{i}^{s} \times\right.\right. \\
& \left.\frac{1}{(2 i+1) \tilde{\alpha}_{3}} \cos (2 i+1) g\right)+L^{4}\left[A_{1} \sum_{i=-2}^{2^{\prime}} \sum_{j=0}^{2^{\prime}} \frac{1}{j \tilde{\alpha}_{1}+2 i \tilde{\alpha}_{3}}\left(\tilde{p}_{i j}^{c} \sin (2 i g+j h)-\tilde{p}_{i j}^{s} \cos (2 i g+j h)\right)\right. \\
& +A_{3} \sum_{i=-2}^{2} \sum_{j=-1}^{1} \sum_{m=0}^{2} \frac{1}{2\left(m \tilde{\alpha}_{1}+j \tilde{\alpha}_{2}+i \tilde{\alpha}_{3}\right)}\left(\tilde{\tilde{p}}_{m i j}^{c} \sin 2(i g+m(h+\Gamma)+j k)\right. \\
& \left.\left.-\tilde{\tilde{p}}_{m i j}^{s} \cos 2\left(i g+m(h+\Gamma)+j k_{\Theta}\right)\right)\right\}+\frac{2}{3}\left\{\sum _ { z \geq 0 } \sum _ { j = - 2 } ^ { 2 ^ { \prime } } \sum _ { i = - 1 } ^ { 1 ^ { \prime } } \frac { 1 } { ( j + 2 i ) \tilde { \alpha } _ { 3 } } \left(\tilde{W}_{z j i}^{c} \sin (j+2 i) g\right.\right. \\
& \left.-\tilde{W}_{z i j}^{s} \cos (j+2 i) g\right)+A_{1} \sum_{z=0}^{3^{\prime}} \sum_{j=-2}^{2^{\prime}} \sum_{i=0}^{2^{\prime}} \sum_{n=-1}^{1} \frac{1}{i \tilde{\alpha}_{1}+(2 j+2 n) \tilde{\alpha}_{3}}\left(\tilde{\lambda}_{z i j n}^{c} \sin (2(j+n) g+i h)\right. \\
& \left.-\tilde{\lambda}_{z i j n}^{s} \cos (2(j+n) g+i h)\right)+A_{3} \sum_{z=0}^{3^{\prime}} \sum_{j=-2}^{2^{\prime}} \sum_{i=-1}^{1^{\prime}} \sum_{n=-1}^{1^{\prime}} \frac{1}{2\left(\tilde{\alpha}_{1}+i \tilde{\alpha}_{2}+(j+n) \tilde{\alpha}_{3}\right)}\left(\tilde{\tilde{\lambda}}_{z i j n}^{c} \sin 2((j+n) g\right. \\
& \left.\left.+h+\Gamma+i k_{\Theta}\right)-\tilde{\tilde{\lambda}}_{z i j n}^{s} \cos 2\left((j+n) g+h+\Gamma+i k_{\Theta}\right)\right)+A_{3} \sum_{z=0}^{3^{\prime}} \sum_{i=0}^{1^{\prime}} \sum_{j=-2}^{2^{\prime}} \frac{1}{2\left(i \tilde{\alpha}_{2}+j \tilde{\alpha}_{3}\right)}\left(\tilde{\omega}_{z i j}^{c} \times\right.
\end{aligned}
$$
zero divisors are to be excluded. tively.

$W_{3}^{* * *}=0$

and $\frac{1}{2} \Pi_{00}\left(\frac{h}{\tilde{\alpha}_{1}}+\frac{g}{\tilde{\alpha}_{3}}\right), \frac{1}{3} \tilde{\Pi}_{000}\left(\frac{g}{\tilde{\alpha}_{3}}+\frac{h}{\tilde{\alpha}_{1}}+\frac{k_{\Theta}}{\tilde{\alpha}_{2}}\right)$ and $\chi_{o}^{c} \frac{g}{\tilde{\alpha}_{3}} \quad$ respec-

The $\mathrm{z}$ over the summation signs indicates that terms with

where the prime over the summation signs indicates that the sine terms are to be replaced in succession by 


$$
\begin{aligned}
& \left.\sin 2\left(j g+i k_{\Theta}\right)-\tilde{\omega}_{z i j}^{s} \cos 2\left(j g+i k_{\Theta}\right)\right)-A A_{3} \sum_{i=-1}^{1^{\prime}} \sum_{j=-1}^{1^{\prime}} \frac{1}{2\left(\tilde{\alpha}_{1}+i \tilde{\alpha}_{2}+j \tilde{\alpha}_{3}\right)} \tilde{\tilde{\omega}}_{i j} \sin 2(j g+h \\
& \left.\left.+\Gamma+i k_{\Theta}\right)\right\}+\frac{1}{15}\left\{-\sum_{i=0}^{2^{\prime}} \sum_{j=-1}^{1^{\prime}} \frac{\tilde{\alpha}_{4 i j}}{i \tilde{\alpha}_{1}+2 j \tilde{\alpha}_{3}}\left[\left(2 A_{1}^{2} \tilde{\alpha}_{82}+2 A_{1} A_{3} \tilde{\alpha}_{83}\right) j+A_{1}^{2} \tilde{\alpha}_{84} i\right] \sin (2 j g+i h)\right. \\
& -2 \sum_{m=0}^{1^{\prime}} \sum_{i=-1}^{1^{\prime}} \sum_{j=-1}^{1^{\prime}} \frac{\tilde{\alpha}_{5 m i j}}{2\left(m \tilde{\alpha}_{1}+i \tilde{\alpha}_{2}+j \tilde{\alpha}_{3}\right)}\left[\left(A_{1} A_{3} \tilde{\alpha}_{82}+A_{3}^{2} \tilde{\alpha}_{83}\right) j+A_{1} A_{3} \tilde{\alpha}_{84} m\right] \sin 2[j g+m(h \\
& \left.\left.+\Gamma)+i k_{\Theta}\right]-\frac{1}{\tilde{\alpha}_{3}}\left(A_{1} \tilde{\alpha}_{82}+A_{3} \tilde{\alpha}_{83}\right)\left(\tilde{\alpha}_{61}^{s} \sin 2 g+\tilde{\alpha}_{61}^{c} \cos g\right)\right\}+\frac{1}{30} \sum_{i=1}^{4^{\prime}}\left[\tilde{J}_{0}^{i}+\tilde{\rho}_{0}^{i}\right. \\
& \left.+A_{1}\left(\tilde{J}_{1}^{i}+\tilde{\rho}_{1}^{i}\right)+A_{1} A_{3}\left(\tilde{J}_{13}^{i}+\tilde{\rho}_{13}^{i}\right)+A_{1}^{2}\left(\tilde{J}_{1}^{i}+\tilde{\rho}_{1}^{i}\right)+A_{3}\left(\tilde{J}_{3}^{i}+\tilde{\rho}_{3}^{i}\right)+A_{3}^{2}\left(\tilde{J}_{33}^{i}+\tilde{\rho}_{33}^{i}\right)\right]
\end{aligned}
$$

\section{THE COMPUTATION OF POSITION AND VELOCITY}

The equations of motion are now reduced to

$\frac{d U^{\prime \prime \prime}}{d t}=-\frac{\partial F^{* * *}}{\partial u^{\prime \prime \prime}}=0 \quad \frac{d u^{\prime \prime \prime}}{d t}=\frac{\partial F^{* * * *}}{\partial U^{\prime \prime \prime}}=$

where 's are arbitrary constant. These equations can be integrated to

$U^{\prime \prime \prime}=U_{0}^{\prime \prime \prime} \quad u^{\prime \prime \prime}=u_{0}^{\prime \prime \prime}+t$

where the constant $\left(u_{0}^{\prime \prime \prime}, U_{0}^{\prime \prime \prime}\right)$ are to be determined from the initial conditions.

Let the elements $\left(u_{0}, U_{0}\right)$ be known at a given initial epoch $t_{0}$ then we can obtain the constants $\left(u_{0}^{\prime \prime \prime}, U_{0}^{\prime \prime \prime}\right)$ as follows:

1) One can compute initial values $\left(u_{0}^{\prime}, U_{0}^{\prime}\right)$ from

$u_{0}^{\prime}=u_{0}+\sum_{n=1}^{5} \frac{\varepsilon^{n}}{n !} u_{0}^{(n)} \quad U_{0}^{\prime}=U_{0}+\sum_{n=1}^{5} \frac{\varepsilon^{n}}{n !} U_{0}^{(n)}$

2) Similarly we can compute the initial values $\left(u_{0}^{\prime \prime}, U_{0}^{\prime \prime}\right)$ from

$u_{0}^{\prime \prime}=u_{0}^{\prime}+\sum_{n=1}^{5} \frac{\varepsilon^{n}}{n !} u_{0}^{\prime(n)} \quad U_{0}^{\prime \prime}=U_{0}^{\prime}+\sum_{n=1}^{5} \frac{\varepsilon^{n}}{n !} U_{0}^{\prime(n)}$

3) Also we can compute the constants $\left(u_{0}^{\prime \prime \prime}, U_{0}^{\prime \prime \prime}\right)$ from

$u_{0}^{\prime \prime \prime}=u_{0}^{\prime \prime}+\sum_{n=1}^{5} \frac{\varepsilon^{n}}{n !} u_{0}^{\prime \prime(n)} \quad U_{0}^{\prime \prime \prime}=U_{0}^{\prime \prime}+\sum_{n=1}^{5} \frac{\varepsilon^{n}}{n !} U_{0}^{\prime \prime(n)}$

Now having determined $U_{0}^{\prime \prime \prime}$ we can evaluate $F^{* * *}=F^{* * *}\left(U^{\prime \prime \prime}\right)$ and in turn the constant $\mathrm{s}$ are now known. Then the position and velocity at any time $t$ can now be computed as follows:

We compute $u^{\prime \prime}=u^{\prime \prime \prime}+\sum_{n=1}^{5} \frac{\varepsilon^{n}}{n !} u^{\prime \prime \prime}(n) \quad U^{\prime \prime}=U^{\prime \prime \prime}+\sum_{n=1}^{5} \frac{\varepsilon^{n}}{n !} U^{\prime \prime \prime}(n)$ and $\quad u^{\prime}=u^{\prime \prime}+\sum_{n=1}^{5} \frac{\varepsilon^{n}}{n !} u^{\prime \prime(n)} \quad U^{\prime}=U^{\prime \prime}+\sum_{n=1}^{5} \frac{\varepsilon^{n}}{n !} U^{\prime \prime(n)}$

finally $\quad u=u^{\prime}+\sum_{n=1}^{5} \frac{\varepsilon^{n}}{n !} u^{\prime(n)} \quad U=U^{\prime}+\sum_{n=1}^{5} \frac{\varepsilon^{n}}{n !} U^{\prime(n)}$

Having determined $u=\left(l, g, h, k_{o}, k_{\Theta}\right)$ and $U=\left(L, G, H, K_{o}, K_{\Theta}\right)$ at time $t$, one can easily compute the position and the velocity.

\section{CONCLUSION}

In this work we developed analytical solution for the combined effects of solar radiation pressure and the gravitation of the Earth, Moon and the Sun on the orbits of high altitude satellites. The mathematical model included the zonal harmonics of the geopotential effects up to $J_{4}$. The equations of motion are derived in terms of a set of ten canonical elements representing the Delaunay variables augmented by the arguments of latitude of the moon and sun and their conjugate momenta to account for the explicit appearance of the time through the motions of the sun and of the moon. The resulting disturbing function is developed in a form suitable to facilitate the subsequent developments to solve the equations of motion. The usefulness of the theory appears in using perturbation techniques based on the LieDeprit- Kamel transform for elimination of the short, intermediate and long-period terms from the Hamiltonian through three canonical transformations.

This technique has many advantages: the perturbation theory is based on explicit transformations; the main part of the development of perturbations is reduced to the evaluation of Poisson brackets, which facilitated the construction of recursive algorithms and made it more access able to be implemented by computers. As a result of the invariance of Poisson brackets under canonical transformations, the generators as well as the Hamiltonian are also invariant, the transformation and its inverse are usually obtained along the same lines and it is possible to give a direct expression for any function of the old variables in terms of the new variables.

Finally a procedure for the computation of the position and velocity at any time is presented.

In conclusion the analytical works are so important for the evaluation of the problems of orbital motions of different 
bodies under the effects of external forces such as drag, radiation pressure...etc.

We believe that the treatments in the analytical models describing the forces and motion can improve the accuracy of the computations and satellite life time.

\section{REFERENCES}

[1] Musen P. The influence of the solar radiation pressure on the motion of an artificial satellite. J Geophys Res 1960; 65:1391.

[2] Kozai Y. Effects motion of an artificial satellite. SAO Spec Rep1961; 56.

[3] Brower D. Dynamic of satellites. In: Roy M, Ed. New York: Academic Press 1963.

[4] Sehnal L. Radiation pressure effects in the motion of artificial satellites. In: Morando B, Ed. Dynamics of satellites. Berlin; New York: Springer-Verlag 1970; pp. 262-2.

[5] Brookes CJ, Ryland FC. The effects of diffuse solar radiation on the motion of an artificial satellite. Celestial Mech 1982;27: 353.

[6] Krivov AV, Getino J. Orbital evolution of high-altitude balloon satellites. Astron Astrophys J 1997; 318: 308.

[7] Cook DG. Solar radiation pressure modeling issues for high altitude satellite. Master's Thesis AD-A390187 Graduate School of Engineering and Management 2001.

[8] El-Saftawy MI. Analytical study of the resonance caused by solar radiation pressure on a spacecraft. Astrophys Space Sci 2005; 295: 407-19.

[9] Abd El-Salam FA. Perturbative effects on a Mercurian orbiter due to the solar radiation pressure, solar wind and the coronal mass ejections. New Astron 2007; 12: 490-6.

[10] Kampos B. Guidance flight mechanics and trajectory optimization. Nasa CR-1008, 1968; Vol. IX.
[11] Kozai Y. A New method to compute lunisolar perturbations in satellite motions. SAO Spec Report 349, 1973.

[12] Giacaglia GEO. Lunar perturbations of artificial satellites of the earth. Celestial Mech 1974; 9: 239.

[13] Richardson DL. In: Nacozy P and Sylvio Ferraz- Mello, Eds. Natural and artificial satellite motion. International Symposium for Natural and Artificial Satellite Motion. Austin: University of Texas Press 1979.

[14] Lane MT. On analytic modeling of lunar perturbations of artificial satellites of the earth. Celest Mech Dyn Astron 1989; 46: 287.

[15] Radwan M. Resonance caused by the Luni-Solar attractions on a satellite of the oblate earth. Astrophys Space Sci 2002; 282: 55162.

[16] Wnuk E. Orbital evolution of space debris. Adv Space Res 2001; 28: $1397-1402$

[17] Wnuk E, Golebiewska J. The relative motion of earth orbiting satellites. Celestial Mech Dyn Astron 2005; 91: 373-89.

[18] Kaula WH. Development of the lunar and solar disturbing functions for a close satellite. Astron J 1962; 67: 300.

[19] Sehnal L. In: Giacaglia T, Ed. Satellite dynamics. Berlin: New York: Springer-Verlag 1975.

[20] Anselmo L, Bertotti B, Farinella P, Milani A, Nobili AM. Orbital perturbations due to radiation pressure for a spacecraft of complex shape. Celestial Mech 1983; 29: 7.

[21] Saad NA. Analytical development for the combined solar radiation pressure and luni-solar effects on the orbit of high altitude earth satellites. Bull Fac Sci Phys Sci Cairo Univ 2007; 75(A): 145-60.

[22] Deprit A. Canonical transformations depending on a small parameter. Celestial Mech 1969; 1: 12.

[23] Kamel AA. Expansion formulae in canonical transformations depending on a small parameter. Celestial Mech 1969; 1: 190.

[24] Fitzpatrick PM. Principles of celestial mechanics. New York: Academic Press 1970.

[25] Hori G. Space mathematics. Am Math Soc 1966; 1(3): 167.

Received: February 2, 2010

(C) Saad et al.; Licensee Bentham Open.

This is an open access article licensed under the terms of the Creative Commons Attribution Non-Commercial License (http://creativecommons.org/licenses/by-nc/3.0/) which permits unrestricted, non-commercial use, distribution and reproduction in any medium, provided the work is properly cited. 